\title{
Kartagener's Syndrome Presenting As Bilateral Recurrent Nasal Polyposis In A Young Boy
}

Zeba Ahmed, Warda Waseem, Uroosa Saman

\author{
ABSTRACT:
}

Kartagener's syndrome is a very rare congenital disease consists of a classic triad, sinusitis, situs inversus and bronchiectasis. Approximately one half of patients with primary ciliary dyskinesia have situs inversus and Kartagener syndrome. We are presenting a case of Kartagener's syndrome in a 10-year-old boy presented with chronic sinusitis leading to bilateral multiple nasal polyposis. He also had situs inversus and chronic bronchiectasis. He had undergone surgery two years back for nasal polyposis but now again presenting as recurrent nasal polyposis. In order to prevent the dreadful complications correct diagnosis in early life is very important in such patients.

Keywords: sinusitis, primary ciliary dyskinesia, Kartagener's syndrome, situs inversus Bronchiectasis

\section{INTRODUCTION:}

Kartagener syndrome $(\mathrm{KS})$ is an inherited autosomal recessive disorder. It causes defects in the action of ciliary movement, comprises of triad sinusitis, situs inversus and bronchiectasis. It was first described by Siewert1 and recognized as a congenital syndrome by Manes Kartagener in $1933^{2}$. These patients usually present with recurrent respiratory infections and infertility in adulthood. Prevalence of Kartagener syndrome is $1 ; 20,000-1: 30,000^{3}$. We are presenting a case of Kartagener's syndrome in a 10 years old boy presented with chronic sinusitis leading to bilateral multiple nasal polyposis.

\section{CASE REPORT:}

A 10-year-old boy presented in outpatient department of Civil Hospital Karachi with bilateral nasal obstruction, rhinorrhea, sneezing, post-nasal dripping off and on since childhood. There was also impaired sense of smell and mild hearing loss on left side. There was no history of allergy, sleep apnea and weight loss but there was a history of fever, cough and recurrent chest infections in past. He also had history of surgical operation for nasal polyposis 2 years back.

On examination, he was a young boy of average built and height, afebrile, conscious and well oriented with no signs of pallor, icterus, cyanosis, clubbing, edema and raised JVP. Bilateral cervical lymph nodes were not palpable. While

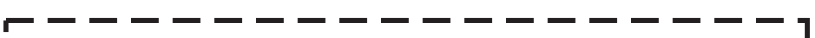

I Zeba Ahmed,

Department of Otorhinolaryngology - Head and Neck Surgery,

I Dow Medical College,

I Dow University of Health Sciences and Civil Hospital Karachi.

Email: ahmed.khanz@hotmail.com

I Warda Waseem,

Department of Otorhinolaryngology - Head and Neck Surgery,

Dow Medical College,

I Dow University of Health Sciences and Civil Hospital Karachi.

I Uroosa Saman

I Department of Otorhinolaryngology - Head and Neck Surgery,

Dow Medical College,

I Dow University of Health Sciences and Civil Hospital Karachi. I

Received: 03-09-2018

Accepted: 26-09-2018

10 examining the nose, no obvious external nasal deformity was observed but there was slight widening of the nasal bridge (fig. 1). Anterior rhinoscopy showed bilateral, multiple, glistening, polypoidal masses in the nasal cavity, which do not bleed on touch. Nasal patency was almost absent on both sides with loss of sense of smell. Oral and ear examination was insignificant except some retraction of the tympanic membrane on the left side. On chest auscultation, there was no added sound on both sides. Heart sound could not appreciate on left side but rather present on the right side, with apex beat was palpable on $5^{\text {th }}$ right inter coastal space. Rest of the examination was insignificant.

Plain x-ray of the nose and paranasal sinuses (Water's view) was done showing soft tissue opacity in both nasal cavities, maxillary sinuses, frontal sinuses and sphenoid sinuses on both sides (fig. 2). Plain X-ray chest (PA view) was done showing dextrocardia and bronchiectasis changes in both the lungs (fig. 3). CT scan of the nose and paranasal sinuses with contrast was done which revealed bilateral nasal polyposis along with moderate enlargement of the adenoids and some deviated nasal septum (fig 3 and 4). Ultrasonography of the whole abdomen was done which confirmed situs inversus and left kidney was distorted and shows loss of cortico-medullay junction and hydronephrosis. Pure tone audiogram showed mild conductive deafness in the left ear with normal hearing in the right ear and tympanogram showed type $\mathrm{C}$ curve on the left side and type A curve on the right side. Hematological investigations showed hemoglobin $10.5 \mathrm{gm} / \mathrm{dl}$, total leucocyte count of $9.5 \times 10^{3} / \mathrm{ml}$ and slightly raise platelet count of $529 \times 10^{3} / \mathrm{ml}$. Erythrocyte sedimentation rate (ESR) and serum electrolytes were also normal in range. Sweat test for chloride was also undertaken to rule out cystic fibrosis and it was negative. We planned surgical treatment for the recurrent nasal polyposis and endoscopic sinus surgery was performed bilaterally. Multiple nasal polypi were noticed bilaterally in the nasal cavity, maxillary, ethmoidal, frontal and sphenoidal sinuses along with frank pus at the antrum of left maxillary sinus which was removed. As it was the revision surgery on this patient, most of the land mark were not identifiable. After 
surgery he was advised for influenza vaccine and discharged on antibiotics, antihistamine and nasal decongest and steroidal sprays and called for regular follow up in ENT OPD. He was also referred to nephrology department for kidney related issue. During the follow-up period of 8 months, patient was alright regarding his nasal problem.

\section{DISCUSSION:}

Primary ciliary dyskinesia (PCD), also called as immotile ciliary syndrome or Kartagener's syndrome is a rare, ciliopathic autosomal recessive genetic disorder that causes defects in the action of cilia lining of the respiratory tract (both lower and upper, including sinuses, Eustachian tube and middle ear), fallopian tubes in female, and flagella of the sperm in the males ${ }^{4}$. It is defined as the combination of sinusitis, situs inversus, bronchiectasis and otitis media. Pseudostratified ciliated columnar cells that line the nasopharynx, middle ear, paranasal sinuses, larynx, trachea

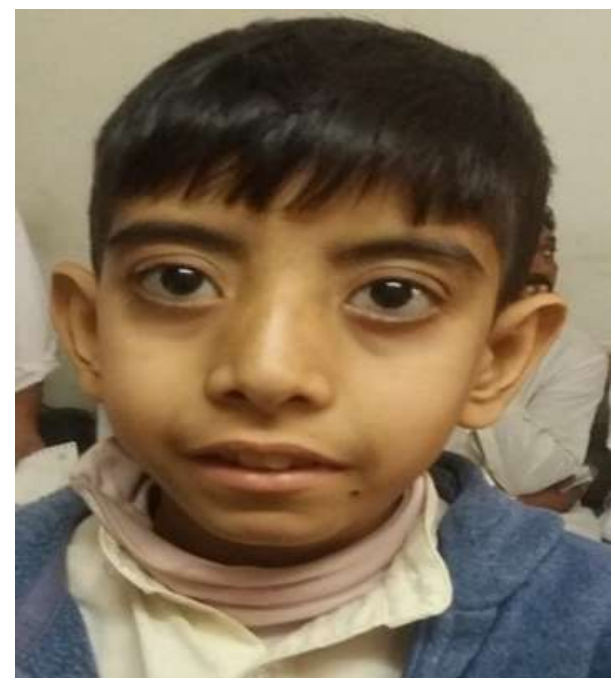

Fig. 1 Patient with Kartagener's Syndrome (Reproduced with written permission of the patient's parents)

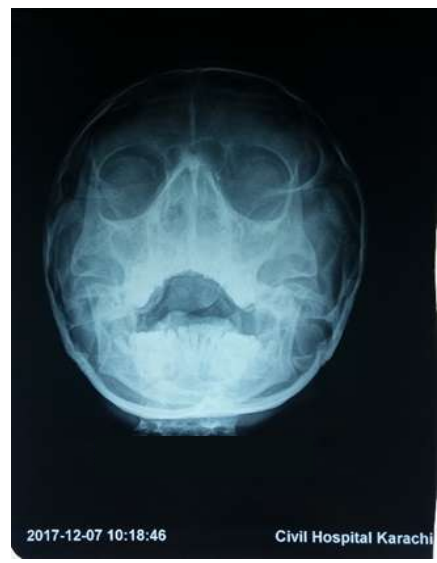

Fig.2 Plain X ray of the nose and PNS showing opacities in bilateral nasal cavity and sinuses

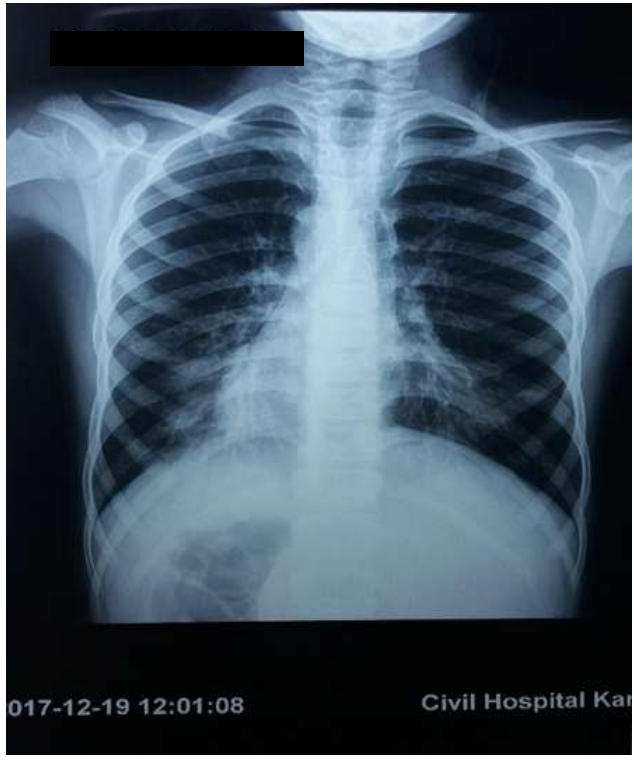

Fig.3 X Ray Chest P.A view showing cardiac shadow on the right side and bronchiectasis changes in the lungs

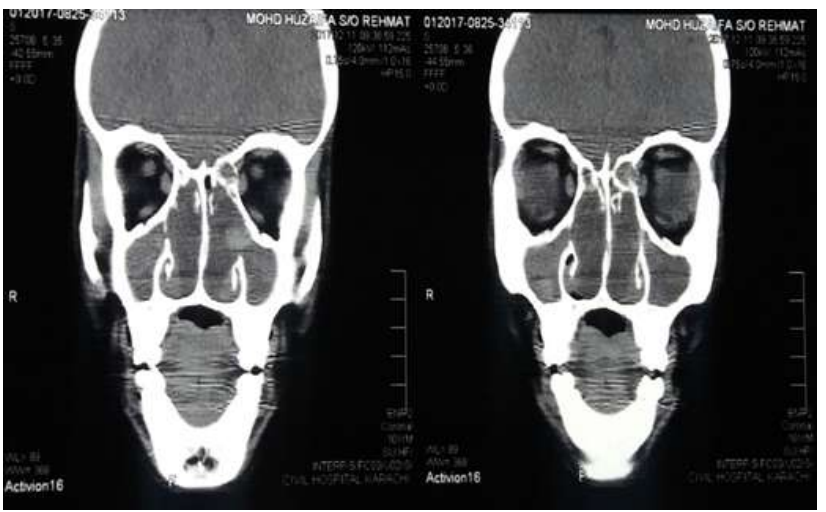

Fig.4 CT Scan of the Nose and PNS coronal view showing homogenous opacities in the nasal cavities and maxillary sinuses on both sides.

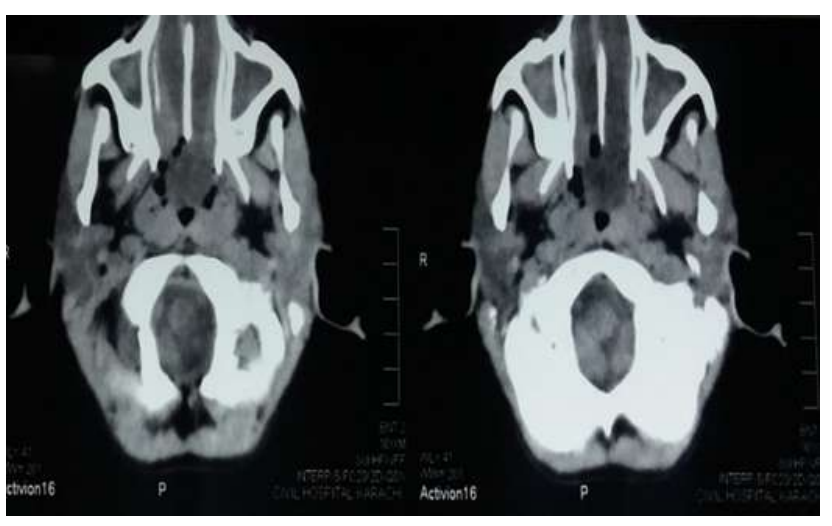

Fig.5 CT Scan of the nose and PNS in axial view showing similar findings 
and bronchi are affected. Functional disturbance of the muco-ciliary clearance due to uncoordinated and ineffective ciliary movement can progress to long standing sino-nasal, aural, and pulmonary problems. More than 200 proteins and polypeptides are engaged in ciliary formation ${ }^{5}$. Any abnormality involving these structures may impair ciliary function.

PCD is a genetically heterogenous disorder affecting motile cilia which are made up of approximately 250 protein $^{6}$. Around $90 \%$ of individuals with PCD have ultrastructural defects affecting proteins in the outer and/or inner dynein arm which give cilia their motility, with roughly $38 \%$ of these defects caused by mutations on two genes DNAI1 and DNAH5, both of which code for protein found in outer ciliary dynein $\mathrm{arm}^{7}$.

Clinical signs and symptoms can be tracked back to early childhood. There is usually a wide range of defects in ciliary ultrastructure and motility which impairs muco-ciliary clearance and thus causes sinopulmonary symptoms with differing severity. Nasal manifestations are chronic rhinitis or sinusitis and nasal polyposis, nasal obstruction and rhinorrhea. Sinusitis in Kartagener's syndrome is the least characteristic feature and can coexist with the absence or hypoplasia of one or more sinuses, nasal polyposis or sinus infection $^{8}$. Ear problems includes otitis media with effusion, and recurrent otitis media. Chronic productive cough and recurrent pulmonary infections are the usual chest complaints. Hemoptysis may also be present. Dyspnea or wheezing usually indicates either widespread bronchiectasis changes or underlying chronic obstructive pulmonary disease.

Normal ciliary beating is also necessary for visceral rotation and orientation during embryonic development. Patients with KS may have either situs solitus i.e., dextrocardia only or situs inversus totalis, where all the visceral structures are on the opposite side ${ }^{9}$. Delay in diagnosis leads to progression of KS with complications i.e. bronchiectasis, pulmonary fibrosis, and finally impaired lung and heart function ${ }^{10}$. KS is often diagnosed incidentally on routine radiological examination, of chest $\mathrm{X}$-ray that shows dextrocardia and situs inversus is on routine checkup. Computed Tomography (CT) of thorax, further shows malrotation or bronchiectasis changes and ultra-sonogram of abdomen reveal situs inversus totalis. CT of paranasal sinuses defines pan-sinusitis, polyposis or hypoplasia of sinuses helping in early diagnosis of disease. Audiometry and tympanometry aid in identifying conductive hearing loss and presence of fluid in the middle ear. Spirometry studies help to evaluate the severity of pulmonary involvement. Semen analysis for sperm motility and ultra-structure is helpful regarding fertility issues. Male patients with KS invariably are infertile, whereas females usually have reduced fertility ${ }^{11}$. Due to the absence of microscopic facility at every hospital, leaves the diagnosis to be made exclusively on clinical examination accompanied with imaging studies.
Anticipated treatment is required to relieve symptoms and to avoid irreversible complications. It comprises of medication, surgery, and some adjuvant therapies. For acute bacterial exacerbations and prophylaxis, antibiotics are indicated. Bronchodilators, inhaled corticosteroids, mucolytic agents, pneumococcal and influenza immunization and chest physiotherapy and positive expiratory pressure devices are recommended and are beneficial. Surgical treatment like functional endoscopic sinus surgery (FESS) is helpful in patients with nasal polyposis and chronic rhino sinusitis. A consistent postoperative follow is very important in preventing recurrence of disease and to reduce morbidities due to retained secretions, impaired muco-ciliary clearance and susceptibility to chronic recurrent airway infections. Lobectomy and pneumonectomy are recommended for localized lung damage with recurrent hemoptysis or respiratory exacerbations. Infertile patients can be treated by advanced micromanipulation techniques and by in vitro fertilization techniques ${ }^{12}$. Genetic testing carries the immense future potential for developing gene therapy and genetic counselling.

\section{CONCLUSION:}

Kartagener's syndrome is a very rare condition. Clinical follow-up at regular intervals is very important in these patients to prevent complications. It may exhibit variable and atypical clinical presentations and severity due to its multi-system involvement and reverse positioning of internal organs.

\section{REFERENCES:}

1. Berdon WE, McManus C, Afzelius B. More on Kartagener's syndrome and the contributions of Afzelius and A.K. Siewert. Pediatr Radiol 2004; 34(7): 585-6

2. Taušan $\mathrm{Dj}$, et al. Kartagener's syndrome: A case report. Vojnosanit Pregl 2016; 73(9): 873-876.

3. Blouin JL, Meeks M, Radhakrishna U, et al. Primary ciliary dyskinesia: a genome-wide linkage analysis reveals extensive locus heterogeneity. Eur J Hum Genet. 2000;8(2):109-118

4. Chodhari,R;MITCHISON.H;M;Meek,M(2004)"cilia ,primary ciliary dyskinesia and molecular genetics"peadriatic respiratory review.5(1)69-76

5. Cowan MJ, Gladwin MT, Shelhamer JH. Disorders of ciliary motility. Am J Med Sci. 2001; 321:3-10.

6. Zariwala MA, Knwles MR, Leigh MW. Primary ciliary dyskinesia. Gene reviews (Internet). 2007;https://www.ncbi. nlm.nih.gov/books/NBK1122/

7. Zariwala MA, Knowles MR, Omran H. Genetic defect in ciliary structure and function. Annual review of physiology 2007; 69: 423-50.

8. Safian LS, Mandeville FB. Kartagener's syndrome in identical male twins and a female sibling: report of cases with comments on pathology and familial manifestation. J Florida Med Assoc. 1959;45:1143-1148.

9. None PG, Bali D, Carson JL, et al. Discordant organ laterality in monozygotic twins with primary ciliary dyskinesia. Am J Med Genet. 1999;82:155-160. 
Kartagener's Syndrome Presenting As Bilateral Nasal Polyposis In A Young Boy

10. Tang X, Zou J, Liu S, et al. Endoscopic sinus surgery for treatment of Kartagener syndrome: a case report. Balkan MedJ. 2013;30:244-247

12. Kay VJ, Irvine DS. Successful in-vitro fertilization pregnancy with spermatozoa from a patient with Kartagener's syndrome: case report. Hum Reprod. 2000; 15:135-138.

11. Afzelius BA, Biasson R. Male and female infertility problems in the immotile cilia syndrome. Eur J Respir Dis Suppl. 1983;127:144-147.

$\bullet \bullet \bullet \hat{\gamma} \bullet \bullet \bullet$ 